\title{
Co-culture of Umbilical Cord Mesenchymal Stem Cells and Bovine Mammary Epithelial Cells Restrains Apoptosis
}

\author{
Wei Shao ${ }^{1}$, Yankun Zhao ${ }^{2}$, Hao Zhang ${ }^{3}$, Liwen Wang ${ }^{1}$, Kaile Wu ${ }^{1}$, Chenglong Luo ${ }^{1}$, Donghai Wang ${ }^{1}$, Liyun \\ $\mathrm{Xu}^{1}$ and Xiong $\mathrm{Yu}^{1 *}$ \\ ${ }^{1}$ Xinjiang Agricultural University, China \\ ${ }^{2}$ Xinjiang Academy of Agriculture Science, China \\ ${ }^{3}$ Yili Vocational and Technical College, China
}

*Corresponding author: Xiong Yu, Xinjiang Agricultural University, China

\begin{tabular}{l}
\hline ARTICLE INFO \\
\hline Received: 慧 January 29, 2020 \\
Published: 㓞 February 06, 2020
\end{tabular}

Citation: Wei Shao, Yankun Zhao, Hao Zhang, Liwen Wang, Kaile Wu, Chenglong Luo, Donghai Wang, Liyun Xu, Xiong Yu. Co-culture of Umbilical Cord Mesenchymal Stem Cells and Bovine Mammary Epithelial Cells Restrains Apoptosis. Biomed J Sci \& Tech Res 25(3)-2020. BJSTR. MS.ID.004192.

Abbreviations: UC-MSCs: Umbilical Cord Mesenchymal Stem Cells; IGF-I: Insulin Like Growth Factor-I; DMEM-F12: Dulbecco's Modified Eagle Medium-F12; FBS: Fetal Bovine Serum

\begin{abstract}
The goal of the present study is to investigate the capacity of co-cultured Umbilical Cord Mesenchymal Stem Cells (UC-MSCs) and BMECs to restrain BMECs apoptosis. In the current study, the Insulin Like Growth Factor-I (IGF-I) expression, cell proliferation, and apoptosis-related gene expression were evaluated between monoculture and co-culture systems. Meanwhile, the signal pathways related to apoptosis were examined to analyze the potential anti- apoptotic effects that co-culturing with UC-MSCs has on BMECs. Results showed that Co-culture of BMECs with UC-MSCs can significantly promote IGF-I secretion and BMEC proliferation in comparison to that of BMECs in monocultures. UCMSCs can effectively restrain BMECs apoptosis by reactivating IGF-I through the PI-3K/ AKT/mTOR signaling pathway, as well as the JAK/STAT pathway. Herein, it was found that IGF-I can up-regulate the expression of the anti-apoptotic geneBcl-2 and down-regulate the expression of the pro-apoptotic genes Caspase- 3 and Bax, effectively restraining BMEC apoptosis. This study demonstrates that a co-culture BMEC/UC-MSC system is a promising strategy for simultaneously restraining BMEC apoptosis while promoting their proliferation in a more effective manner than exogenous supplementation of IGF-I alone. Furthermore, the co-culture system described herein has potential utility for other future animal husbandry applications as well.
\end{abstract}

Keywords: Umbilical Cord Mesenchymal Stem Cells; Bovine Mammary Epithelial Cells; Insulin Like Growth Factor-I; Lactogenic Capacity; Co-Culture

\section{Introduction}

The aim of the present study is to investigate the potential antiapoptotic effects on BMECs which result from their interactions with UC-MSCs in co-culture. Specifically, the relevant anti-apoptotic mechanism was evaluated by exploring the underlying signaling pathways. For this purpose, IGF-I/ IGF-IR expression, as well as the expression profiles of both proliferation and apoptosis-related genes were examined and compared between mono- and coculture systems. In particular, the signaling pathways associated with apoptosis were analyzed to clarify the anti- apoptosis effect of co-culture on BMECs.

\section{Materials and Methods}

\section{Cell Culture}

Holstein-Friesian UC-MSCs were isolated and identified according to our previous work [1]. Briefly, UC-MSCs were subcultured in Dulbecco's Modified Eagle Medium: Nutrient Mixture F12 (DMEM-F12) supplemented with FBS (15\%,v/v) and penicillin-streptomycin $(1 \%, v / v)$. Holstein-Friesian BMECs were purchased from Jennio biological technology (Guangzhou, China) and subcultured in RPMI 1640 Medium supplemented with Fetal 
Bovine Serum (FBS) (15\%, v/v), and penicillin-streptomycin $(\mathrm{p} / \mathrm{s}, 1 \%, \mathrm{v} / \mathrm{v})$. Cells at passage 5-8 were used in the subsequent experiments in order to ensure the cell bioactivity. The media and supplements used in the experiments were obtained from Gibco (Thermal fisher, USA). All cells were incubated at $37{ }^{\circ} \mathrm{C}, 5 \% \mathrm{CO} 2$, and $95 \%$ relative humidity.

\section{The Effect of Co-Culture on IGF-I/ IGF-IR Expression}

Direct-/ indirect- contact co-culture systems were employed to investigate the effects of UC-MSC/BMEC co-culture on IGF-I/ IGFIR expression, as well as to confirm the primary source of IGF-I. The direct contact co-cultures used the classical mixed culture method and the indirect method utilized Transwell ${ }^{\circledR}$ solution $(0.4 \mu \mathrm{m}$, Corning, USA). Specifically, the co-culture of UC-MSCs and BMECs adopted an optimal cell ratio (UC-MSCs: BMECs $=1: 2$ ) in accordance to our previous experience [1]. In order to investigate the primary source of secreted IGF-I in the cultures, the cells in the insert chamber of the Transwell were collected for detecting the IGF-I/ IGF-IR gene expressions by a Real Time-PCR assay. Total RNA was extracted using RNeasy Mini Kit (Qiagen, USA) per the manufacturer's instructions.

\section{The Effect of UC-MSCs on BMEC Proliferation}

The effect of UC-MSCs on BMEC proliferation was investigated by direct-/indirect- contact culture methods. Briefly, the culture medium of UC-MSCs at exponential phase was collected as conditioned medium. Subsequently, BMECs or UC-MSCs were dissociated with Trypsin-EDTA (0.25\%) followed by suspension with SFM. In the direct culture group, UC-MSCs and BMECs were mixed at the ratio of 1:2 and seeded in the well plates for co-culture. In the indirect culture group, BMECs were cultured with UC-MSCs conditioned medium. The monoculture of UC-MSCs or BMECs was used as control group.

\section{The Effect and Mechanism Of UC-MSCs On BMECs' Apoptosis}

In order to further investigate the potential anti-apoptotic effect of UC-MSCs on BMECs in the co-culture system, multiple inhibitors of IGF-IR and signal pathway were used in the series of experiments.

Experiment Design: UC-MSCs and BMECs were seeded at a density of $1 \times 105$ cells/well (ratio: $1: 2$ ) in the insert and basolateral chambers of Transwell, respectively. AG1024 (Alexis, USA) (inhibitor of IGF-IR), AG490 (Sigma, USA) (inhibitor of JAK2/ STAT5 signal pathway), and LY294002 (Sigma, USA) (inhibitor of $\mathrm{PI} 3 / \mathrm{KAKT} / \mathrm{mTOR}$ signal pathway) were employed for treating the BMECs at concentrations of $10 \mu \mathrm{mol} / \mathrm{L}, 50 \mu \mathrm{mol} / \mathrm{L}$, and $25 \mu \mathrm{mol} / \mathrm{L}$, respectively $[2,3]$. The untreated groups were added with DMSO as a control. The details of the groups are outlined in below. After 24 hours of culturing, the supernatants and cells of various groups were collected for biochemical analysis. Control group: BMECs; Experimental group: BMECs/UC-MSCs; Treatment I group: BMECs+AG1024;
Treatment II group: (BMECs+AG1024)/UC-MSCs;

Treatment III group: BMECs+AG490;

Treatment IV group: (BMECs+AG490)/UC-MSCs;

Treatment V group: BMECs+AG490+AG1024;

Treatment VI group: (BMECs+AG490+AG1024)/UC-MSCs;

Treatment VII group: BMECs+LY294002;

Treatment VIII group: (BMECs+LY294002)/UC-MSCs;

Treatment IX group: BMECs+AG1024+LY294002;

Treatment X group: (BMECs+AG1024+LY294002)/UC-MSCs.

Apoptosis was examined by flow cytometry [4]. Apoptosis analysis was performed by real-time quantitative PCR. Repetitive ANOVA and SPSS 18.0 software were used to determine statistical significance $(\mathrm{P}<0.05)$ between groups.

\section{Conclusion}

In summary, the UC-MSCs and BMECs co-culture system overcome the challenges and shortcomings of BMEC degeneration and apoptosis in BMECs monoculture. Co-culture of UC-MSCs and BMECs can significantly promote the IGF-I secretion in comparison to that of BMEC monocultures, as the UC-MSCs provide an endogenous source of IGF-I. Moreover, the IGF-I and IGF-IR mRNA expression of BMECs were demonstrably enhanced through co-culture with UCMSCs Additionally, UC-MSCs have been found to promote BMEC proliferation within the co-culture system, with directly co-cultured cells having higher proliferation rates than those that have been co-cultured indirectly. This observable phenomenon is suggested to have a positive correlation with IGF-I expression and secretion. Furthermore, UC-MSCs can effectively restrain BMEC apoptosis by reactivating IGF-I through the PI-3K/AKT/mTOR and JAK/STAT signaling pathways. The ultimate mechanism of protective action that UC-MSCs have on BMECs likely involves the up-regulation of the expression of the BMEC anti-apoptotic gene Bcl-2 and the downregulation of thee pro-apoptotic genes Caspase- 3 and Bax. This study has demonstrated that BMEC/UC-MSC co-culture of system is a promising approach to restrain BMEC apoptosis while promoting their differentiation and proliferation in a more effective manner than what is possible with current techniques which rely solely on exogenous supplementation of BMEC cultures with IGF-I.

\section{Ethics Approval and Consent to Participate}

not applicable

\section{Consent for Publication}

not applicable

\section{Availability of Data and Material}

All data generated or analyzed during this study are included in this published article. 


\section{Conflicts of Interest}

The authors declare no competing financial interests.

\section{Funding}

The funding support from National Natural Science Foundation of China (31560645) and Key Laboratory for Collaborative Center of the Xinjiang Uygur Autonomous Region (2015KL018).

\section{Author Contributions}

WS, YK Z and HZ conceived and supervised the study. WS, YK $\mathrm{Z}$ and LW $\mathrm{W}$ designed the experiments. WS, LW W, KL W and LY X performed the experiments. WS, DH W and CL L analyzed the data. WS and YX revised the paper.WS wrote the paper.

\section{Acknowledgment}

The authors thank the financial support from National Natural Science Foundation of China (31560645) and Key Laboratory for Collaborative Center of the Xinjiang Uygur Autonomous Region (2015KL018).

ISSN: 2574-1241

DOI: $10.26717 /$ BJSTR.2020.25.004192

Xiong Yu. Biomed J Sci \& Tech Res

(C) (P) This work is licensed under Creative Submission Link: https://biomedres.us/submit-manuscript.php

\section{Appendix A. Supplementary Data}

Supplementary data associated to this article can be found online.

\section{References}

1. Zhao Y, Shao W, Luo C, Wu K, Yu X (2017) Co-culture with umbilical cord mesenchymal stem cells promotes the synthesis and mechnism of milk protein in bovine mammary epithelial cells. Xi Bao Yu Fen Zi Mian Yi Xue Za Zhi 33(2): 185-189.

2. Kogai T, Ohashi E, Jacobs MS, Sajid-Crockett S, Fisher ML, et al. (2008) Retinoic Acid Stimulation of the Sodium/Iodide Symporter in MCF7 Breast Cancer Cells Is Meditated by the Insulin Growth Factor-I/ Phosphatidylinositol 3-Kinase and p38 Mitogen-Activated Protein Kinase Signaling Pathways. The Journal of Clinical Endocrinology and Metabolism 93(5): 1884-1892.

3. Lescarbeau RM, Seib FP, Prewitz M, Werner C, Kaplan DL (2012) In Vitro Model of Metastasis to Bone Marrow Mediates Prostate Cancer Castration Resistant Growth through Paracrine and Extracellular Matrix Factors. PLOS ONE 7(8): e40372.

4. Zhou X, Chen L, Wang A, Ma Y, Zhang H, et al. (2015) Multifunctional fluorescent magnetic nanoparticles for lung cancer stem cells research. Colloids Surf B Biointerfaces 134: 431-439.

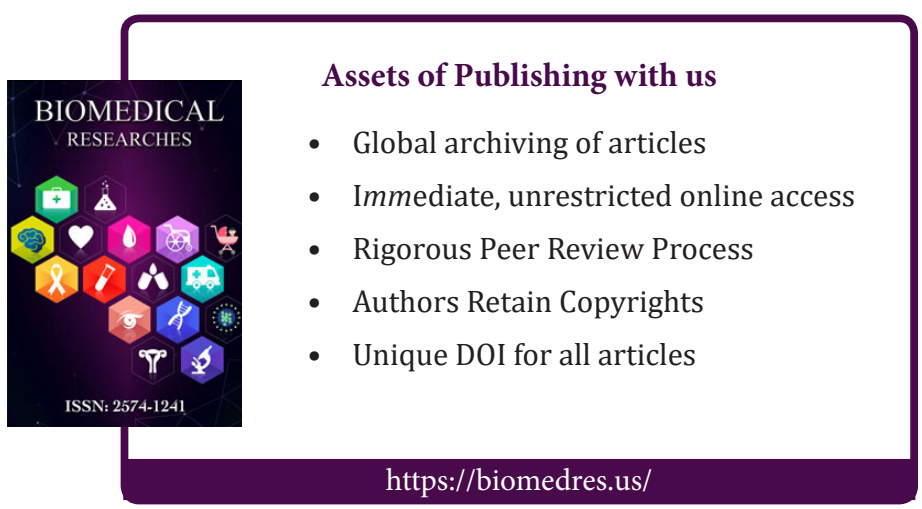

